\title{
Real-time Indoor Patient Movement Pattern Telemonitoring with One-meter Precision
}

\author{
Po-Chou Liang and Paul Krause \\ Department of Computing \\ University of Surrey \\ Guildford, United Kingdom
}

\begin{abstract}
Monitoring of the activities of daily living of the elderly at home is widely recognized as useful for detection of new or deteriorated health conditions. However, the accuracy of existing indoor location tracking systems remains unsatisfactory. The aim of this study was therefore to develop a localization system that can identify a patient's real-time location in a home environment with maximum estimation error of two meters at a $95 \%$ confidence level. A prototype based on a sensor fusion approach was built. This involved the development of both a step detector using the accelerometer and compass of an iPhone 5, and a radiobased localization subsystem using Kalman filter and received signal strength indication (RSSI). The results of our experiments were promising with average estimation error of 0.55 meters. We are confident that with more work our design can be adapted to a home-like environment with a more robust localization solution.
\end{abstract}

Keywords-telemonitoring; movement pattern; localization; location tracking; received signal strength; Kalman filter; step detection; sensor fusion

\section{INTRODUCTION}

For years, there has been a lack of robust evidence for costeffectiveness of remote healthcare systems [1-3]. To address this issue, we set up as our core objective the cost-effective design of real-time home healthcare telemonitoring based on mobile cloud computing. Key considerations about the development of our system included lower cost, reduced intrusiveness, and higher mobility, usability, deployability and portability. A prototype, consisting of three key functional components, including vital sign telemonitoring, safety telemonitoring (mainly on fall detection) and movement pattern telemonitoring, was then developed [4]. The purpose of this paper is to present the design and implementation of our proposed real-time indoor patient movement pattern telemonitoring on the client side, composed of a User Agent module (based on iPhone 5) and a Sensor module (formed by a number of low-cost sensors).

In healthcare, it has been widely acknowledged that inhome monitoring of the elderly or chronic disease outpatients' daily movement patterns is useful for detection of early signs of new or deteriorated health issues. However, to achieve satisfactory accuracy remains a challenge for indoor location tracking/localization [5]. (This paper uses location tracking and localization interchangeably.) According to [6], location tracking applications are divided into two categories, i.e. coarse-grained and fine-grained tracking systems. The former in general detect only the presence of the tracked target, while the latter detect and measure the distance between the target and sensors. For the purpose of this study, we set up our basic requirement as to identify the patient's real-time location within a home environment with maximum estimation error of two meters at a 95\% confidence level. Accordingly, finegrained tracking was designed and implemented in our system.

A radio-based localization technique using trilateration, trigonometry and received signal strength indication (RSSI) from three triangular deployed Bluetooth Low Energy (BLE) sensors was chosen to build the required functionality. However, due to the arbitrary variations of RSSI readings from the sensors in an indoor environment, we found that it is impractical to use raw RSSI data alone to perform reliable realtime estimation of the patient movement. Therefore, to enhance the overall reliability and accuracy of our system, we developed the three following elements: a step detection mechanism (using the accelerometer and compass of an iPhone 5) to produce extra patient location information; a discrete-time Kalman filter to improve distance estimation; and a tight coupling sensor fusion approach to integrate all these features as a whole.

The results of our trials, each performed by a user carrying a User Agent and walking around a small office (54 square meters), were promising with maximum estimation error of 0.81 meters and average estimation error of 0.55 meters in a normal case. To further illustrate both the issues encountered and our solutions to those issues, the remainder of this paper is structured as follows. In the next section, we briefly introduce related work in indoor location tracking and the concepts of RSSI and Kalman filter. In Section III, we describe our design and implementation of our proposed system. Section IV presents the experimental results and our evaluation. Finally, Section V provides our conclusion remarks and future work.

\section{RELATED WORK}

\section{A. Indoor Location Tracking}

Along with the fast-growing smartphone economy, more location-aware or location-based applications have been emerging. For indoor environments, there have been an increasing number of studies on location tracking using wireless signals from neighbouring wireless devices, such as 
Wi-Fi access points and wireless sensor networks (WSN). Among all techniques, the RSSI-based ones have gained popularity in recent years. Nevertheless, as indicated by [5], some major problems in this field remain unresolved, such as computationally intensive and inaccurate localization algorithms, excessive access point installations and unstable wireless signal transmission.

To tackle the problem of unstable wireless signal transmission, both the Monte Carlo [7], [9] and Kalman filter [8], [10] methods were commonly adopted for constructing RSSI-based localization algorithms. This is because both can produce statistically more precise estimation of system states than those solely based on one or a few noisy measurements. However, from our perspective, the need for an extra computer to perform location estimates using the Monte Carlo method, as proposed by some studies [7], [9], raises cost and reliability issues.

Another technique commonly adopted was to produce and store a detailed wireless signal strength map at each specific survey location before performing localization [5], [9], [10]. Location tracking was then conducted by comparing real-time RSSI measurements with the stored signal strength map. However, we consider that the need to produce a wireless signal strength map for each survey location would cause issues not only on system usability, deployability and portability, but also on longer processing time and higher software and hardware requirements, such as storage and database. In some cases, excessive sensor nodes were needed to conduct target tracking. For instance, [8] used both eight static sensor nodes and one or more mobile nodes in its simulations.

With regard to accuracy and performance, some studies could achieve an overall estimation error of about two meters. For example, the distance estimation errors of [5] were within 2.3 meters with $90 \%$ precision, while the average error for walk-through tests of [9] was 2.1 meters. Though the overall distance estimate error of [7] was 1.2 meters, its maximum error was about 3.5 meters and the use of a networked computer for performing heavy computational tasks caused latency of up to eight seconds.

The identified issues mentioned above indicate a remaining gap in achieving wider uptake of indoor location tracking in society. Moreover, lessons learned from these studies suggest that we need to develop a lightweight, but accurate localization algorithm suitable for execution in a smartphone, rather than another computer. We should also avoid the need to produce, store and make comparison of detailed wireless signal maps, as well as the need for excessive hardware installations. These would help achieve higher mobility, reliability, usability, deployability and portability of our real-time telemonitoring system at a lower cost.

\section{B. Received Signal Strength Indication}

Theoretically, this indication is based on the inverse-square law that the wireless signal strength is proportional to the inverse of the square of the distance from the signal source. Equation (1) denotes the relationship between received signal strength and corresponding distance [6]. However, in reality, due to several issues, such as multi-path fading, indoor shadowing, and interference, the relation between signal strength and distance in an indoor environment usually becomes arbitrary. This significantly increases the complexity of RSSI-based distance estimates. To improve the accuracy of RSSI-based location tracking, numerous approaches and algorithms, such as those mentioned in the previous sub-section, have been proposed.

$$
R S S I_{d}=-10 n \log (d)+R S S I_{0}
$$

In (1), $\operatorname{RSSI}_{d}$ (in Decibel-milliwatts) is the RSSI measured at distance $d$ (in meters) from the source; $n$ is the path loss exponent and $\mathrm{RSSI}_{O}$ (in Decibel-milliwatts) is the RSSI measured at one-meter distance.

\section{Kalman Filter}

Because a Kalman filter is relatively lightweight and has a much better convergence rate than a Monte Carlo filter, we chose the former to build our localization algorithm. Equation (2) and (3) explain how to model a system using discrete-time Kalman filter [11]:

$$
\begin{aligned}
& x_{k}=A x_{k-1}+B u_{k}+w_{k} \\
& z_{k}=H x_{k}+v_{k}
\end{aligned}
$$

In (2), $x_{k}$ is the estimate of the system state variable at time $k ; u_{k}$ is the control signal; and $w_{k}$ is the process noise. In (3), $z_{k}$ is the measurement value at time $k$; and $v_{k}$ is the measurement noise. $A, B$ and $H$ are general form matrices, introduced to model the system, and in many cases can be simplified as numeric values. To perform the estimates based on a discretetime Kalman filter, the two sets of equations in Table I can be used repeatedly.

In Table I, $x_{k}^{-}$is the "priori estimate" of the system state at time $k$ before measurement update correction; $x_{k-1}$ is the updated estimate (or posteriori estimate) at time $k-1$ after measurement; $P_{k}^{-}$is the "priori error covariance"; $K_{k}$ is the Kalman Gain; $x_{k}$, which is the updated estimate (or posteriori estimate) at time $k$ after measurement, is the very value we try to find; and $P_{k}$ is the updated error covariance after measurement.

TABLE I. Two SETS of DisCRETE-TIME Kalman Filter EQUations FOR STATE ESTIMATION, AFTER [11]

\begin{tabular}{|l|l|}
\hline $\begin{array}{c}\text { Time Update } \\
\text { (Prediction) Equations }\end{array}$ & \multicolumn{1}{c|}{$\begin{array}{c}\text { Measurement Update } \\
\text { (Correction) Equations }\end{array}$} \\
\hline$x_{k}{ }^{-}=A x_{k-1}+B u_{k}+w_{k}$ & $K_{k}=P_{k}^{-} H^{T}\left(H P_{k}^{-} H^{T}+R\right)^{-1}$ \\
$P_{k}{ }^{-}=A P_{k-1} A^{T}+Q$ & $x_{k}=x_{k}^{-}+K_{k}\left(z_{k}-H x_{k}^{-}\right)$ \\
& $P_{k}=\left(1-K_{k} H\right) P_{k}^{-}$ \\
\hline
\end{tabular}

\section{EXPERIMENTAL DESIGNS}

Our designs and experiments started from the use of raw RSSI data received from three triangular deployed BLE sensors (i.e. TI SensorTags) to estimate the location and movement of a tracked target who held a User Agent (i.e. an iPhone 5) and 
walked around a small office environment (nine meters by six). The three BLE sensors (denoted as $\mathrm{S}_{0}, \mathrm{~S}_{1}$ and $\mathrm{S}_{2}$ ) were placed against three different walls of the office at 1.1 meter height from the floor. However, because of the very diverse and unstable nature of the RSSIs, it was impossible to use the raw RSSI data alone to reliably estimate the location and movement of the target.

We then implemented a Kalman filter to improve RSSI estimates based on noisy RSSI measurements. However, some identified issues in relation to the one-second RSSI update rate implemented by iOS caused the resultant RSSI estimates to become very unreliable. Consequently, we decided to take a sensor fusion approach to perform location estimates. Based on this approach, the design and implementation of a step detector, a Kalman filter, and several estimate optimization mechanisms are described in the following.

\section{A. Step Detector}

A step detector was designed and implemented on the User Agent using accelerometry-related parameters (generated by the iPhone's built-in accelerometer) and heading information (by compass). Two thresholds (i.e. a value of 1.07 gravities followed by a reduction of 0.209 gravities) of the sum vector (SV) of acceleration in X-Y-Z axes were defined. If these thresholds were met during real-time monitoring, the User Agent would signify a detected step when at the same time the Y-axis acceleration was less than or equal to -0.3 gravities or the change of heading angle was less than seven degrees.

Whenever a step or several steps were detected before the User Agent received new RSSI updates from all three reference sensors, a new location of the target, as well as the change of distance between the target and each of the sensors during this period of time, was calculated based on both the pre-defined length of a step (e.g. 0.65 meters) and the heading data.

\section{B. Kalman Filter for Distance Estimates}

A discrete-time Kalman filter was developed for estimating the distance (denoted as $x_{i, k}$; hereafter an added subscript $i$ to each variable shown in Table I refers to sensor $S_{i}$ ) between the target and sensor $S_{i}$ (subscript $i$ could be 0,1 or 2 ) at time $k$. Upon receiving new RSSI updates from a sensor, the distance information calculated by the step detector would be used as the control signal $u_{i, k}$ of the Kalman filter. The reason for waiting for new RSSI updates before starting a new run of state estimation via the Kalman filter was to synchronize the target movement with RSSI readings. This was because the iOS updates RSSI measurements for each sensor at a maximum rate of once per second and updates for different sensors usually occur at different times, while a walking step generally takes less than one second. In the worst case, this synchronization process would incur latency of up to 2.5 seconds.

When a step was correctly detected, the process noise, which occurred mainly due to the inaccuracy of inferring the heading angle and step length, would be relatively small. So we used a value between zero and 0.23 meters (the standard deviation of step detection, if we assumed that on average one in every ten steps was incorrectly detected or missed) as the process noise $w_{i, k}$, depending on how much correlation between the step detector and the Kalman filter was needed. The process noise covariance $\mathrm{Q}_{i}$ was hence equal to $E\left[w_{i, k} w_{i, k}^{T}\right]$.

The value for measurement noise generally became bigger with the increase of distance between the User Agent and sensor $\mathrm{S}_{i}$. Based on our empirical observation, we assumed that the measurement noise $v_{i, k}$ was equal to the converted distance multiplied by a factor of 0.6. Meanwhile, matrices $A, B$ and $H$ in (2) and (3) were all simplified as a numerical constant of one. The initial value of $x_{i, 0}$ was set to a pre-defined distance, as we assumed the real-time monitoring always started at a known location and the error covariance $P_{i, 0}$ was simply set to a nonzero value, e.g. 0.5. With all these considerations and assumptions, the two sets of the Kalman filter equations as shown in Table 1 were ready for estimating the distance between the User Agent and each of the three sensors.

\section{Mechanisms for Estimate Optimization}

To calculate the real-time location of the tracked target based on the outputs from the Kalman filter, traditional techniques, including trilateration, trigonometric functions and Maximum-Likelihood Estimation (MLE) were first adopted. To apply trilateration, the distances from three sensors estimated by the Kalman filter at a certain time were used as radii to create three circles each centered at $S_{0}, S_{1}$ and $S_{2}$ respectively. Ideally, there should have been a joint intersection point among the three circles, representing the optimally estimated real-time location of the target at that precise moment. However, the reality was much more complicated with both underestimates and overestimates of the three distances, probably happening at the same time. Therefore, when there was an area of intersections, rather than a single point, found among the three circles, both trigonometric functions and an iterative procedure based on the MLE were adopted to estimate the target's location. Alternatively, if intersections were found only between two circles or there was not any intersection among the three circles, we enlarged one or two of the circles to create intersections and then applied the techniques mentioned above. However, the resultant estimates were still not satisfactory.

Our assessment revealed that there might be two main causes for such unsatisfactory results. Firstly, the inaccuracy and insufficient number of RSSI measurements had inborn negative impacts on the accuracy of distance estimation. Secondly, the process of manipulating estimated distances to create joint intersections among the three circles had introduced further noises into the system. Consequently, a follow-stepdetection mechanism was developed to address the first issue by choosing a location, nearest to the target location estimated by the step detector rather than based on MLE, within the intersection area. Furthermore, to address these two issues simultaneously, another tight coupling (between step detection and Kalman filter) mechanism was also developed by using the average of distances both estimated by step detection and converted from RSSI readings as the measurement value for the next run of the Kalman filter. This mechanism then chose one existing intersection point nearest to the target location estimated by step detection as the final estimated location of the target at that precise moment. 


\section{PRELIMINARY RESULTS AND EVALUATIONS}

The results of 20 trials on real-time movement pattern monitoring based on our sensor fusion approach varied slightly, mainly due to occasional misestimates of step detection and intermittent drifts of heading outputs generated by the iPhone's compass. After having tuned a number of different parameters, such as path loss exponent and measurement noise, we found that the overall accuracy of our localization algorithm was not significantly affected by these parameters, but mainly by both the accuracy of the step detector, the value of process noise, and our choice of a particular coordinate as our optimal estimate of the real-time target location.

As shown in Table II, both the maximum and average estimate errors based on the tight coupling mechanism are smaller than those based on the other mechanisms. Fig. 1 depicts the results of location tracking in the small office environment based on raw RSSI, the step detection and the tight coupling sensor fusion approach respectively. As these experiments were based on a normal subject/user, we plan to perform further tests and evaluations using a wider range of subjects, including for example Parkinson's disease patients with a tremor issue, in the future.

TABLE II. ESTIMATION ERRORS BASED ON DIFFERENT MECHANISMS

\begin{tabular}{|l|c|c|}
\hline \multicolumn{1}{|c|}{$\begin{array}{c}\text { Mechanisms for Estimate } \\
\text { Optimization }\end{array}$} & $\begin{array}{c}\text { Max. Estimate } \\
\text { Error (meter) }\end{array}$ & $\begin{array}{c}\text { Avg. Estimate } \\
\text { Error (meter) }\end{array}$ \\
\hline Step Detection ${ }^{\text {a }}$ & 1.17 & 0.58 \\
\hline Maximum-Likelihood Estimate & 1.75 & 1.04 \\
\hline Follow-step-detection Mechanism & 1.03 & 0.54 \\
\hline Tight Coupling Mechanism & 0.81 & 0.55 \\
\hline
\end{tabular}

a. excluding results of the worst case

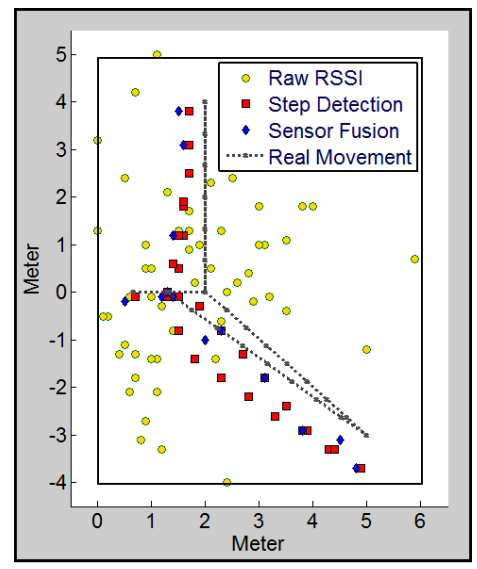

Figure 1. Location tracking based on raw RSSI, step detection and sensor fusion Approach

\section{CONCLUSIONS}

In this paper, we have presented a proposed localization system for real-time indoor patient movement pattern telemonitoring, which is part of our cost-effective design of real-time home healthcare telemonitoring based on mobile cloud computing. To enhance the accuracy of our prototype, we have developed a tight coupling sensor fusion approach and gained promising results. We are confident that with more work our design can be adapted to a home-like environment with a more robust localization solution.

For future work, we plan to integrate accurate location information from force sensitive resistor (FSR) sensors deployed at certain known locations to calibrate the location estimates of our step detector and to improve our overall localization algorithm. In addition, we will adapt our current design to a home-like environment where wireless signal transmission might well be further affected by various floor plans, partition walls and furniture. It is also desirable to further enhance the reliability and accuracy of our step detection algorithm. By doing so, we can use the step detector not only to record where the patient place reference sensors during system set-up and deployment, but also to perform real-time patient movement pattern telemonitoring without the need to implement RSSI-based localization.

\section{REFERENCES}

[1] J. Barlow, D. Singh, S. Bayer and R. Curry, "A systematic review of the benefits of home telecare for frail elderly people and those with longterm conditions", Journal of Telemedicine and Telecare, vol. 13, no. 4, pp. 172-179, Jun. 2007.

[2] T.S. Bergmo, "Economic evaluation in telemedicine - still room for improvement", Journal of Telemedicine and Telecare, Vol. 16, no. 5, pp. 229-331, July 2010.

[3] S. McLean, D. Prott and A. Sheikh, "Telehealthcare for long term conditions", BMJ 2011; 342:d120, doi: 10.1136/bmj.d120, 2011.

[4] P.C. Liang and P. Krause, "Cost-effective design of real-time home healthcare telemonitoring", the 7th International Conference on Health Informatics (HEALTHINF 2014), Angers, France, Mar. 2014.

[5] E. C.L. Chan and G. Baciu, "Introduction to wireless localization", Chapter 1 of "Introduction to wireless localization: with iPhone SDK examples", $1^{\text {st }}$ ed., published by John Wiley and Sons Singapore Pte. Ltd., 2012.

[6] C.C. Pu, C.H. Pu and H.J. Lee, "Indoor location tracking using received signal strength indicator", Chapter 11 of "Emerging communications for wireless sensor networks", published by InTech, 2011

[7] L. Klingbeil and T. Wark, "A Wireless Sensor Network for Real-time Indoor Localization and Motion Monitoring”, 2008 IEEE International Conference on Information Processing in Sensor Networks", Conference Proceedings, pp.39-50, 2008.

[8] S. Zhang et al., "Mobile sensing and simultaneously node localization in wireless sensor networks for human motion tracking", 11th International Conference on Control Automation Robotics \& Vision (ICARCV), Conference Proceedings, pp.2313-2318, 2010.

[9] G.V. Zaruba, M. Huber and F.A. Kamangar, "Indoor location tracking using RSSI readings from a single Wi-Fi access point", published by Springer Science + Business Media, LLC 2006.

[10] M.H. Li, "Precise Indoor Positioning System Using Long Term WLAN Signal Measurement", Master Thesis, Department of Communication Engineering, National Central University, Taiwan, 2010, unpublished.

[11] G. Welch and G. Bishop, "An introduction to the Kalman filter", Tutorial, Department of Computer Science, University of North Carolina at Chapel Hill, 2006, unpublished. 